\title{
El uso de técnicas cuantitativas en la investigación del Derecho y las Ciencias Sociales*
}

\author{
The use of quantitative techniques in law and social sciences research
}

William Guillermo Jiménez**, Orlando Meneses Quintana***

*Producto de la labor investigativa que se desarrolla dentro de los grupos de investigación Teoría del Derecho de la Justicia y de
la Política de la Universidad La Gran Colombia ((proyecto: El desarrollo de la inteligencia artificial y la preservación de derechos
fundamentales en la era digital) y el grupo Política, Derecho y Gestión Pública de la Escuela Superior de Administración Pública.
**Posdoctor en Derecho (Vrije Universiteit Amsterdam), profesor Titular de la Escuela Superior de Administración Pública y la
Universidad La Gran Colombia, miembro de los grupos de investigación Política, Derecho y Gestión Pública (ESAP) y Teoría del
Derecho, de la Justicia y de la Política (UGC), willjime@esap.edu.co, william.jimenez@ugc.edu.co, calle 44 No. 53-37 Bogotá,
Colombia.
***Magíster en Ética y Política (Universidad Nacional), profesor asociado a la Facultad de Derecho de la Universidad La Gran Colombia, grupo de investigación Teoría del Derecho, de la Justicia y de la Política (UGC), orlando.meneses@ugc.edu.co, carrera 5 No.
12B 23, Bogotá, Colombia.

Fecha de recepción: febrero 11 de 2021 Fecha de aprobación: junio 15 de 2021

Para citar este artículo / To reference this article Álvarez, I. (2021) El intelectual universitario censurado en España. Inciso, 23(1) e:1108

DOI: http://dx.doi.org/10.18634/incj.23v.1i.1108

\section{Resumen}

El disminuido uso de las técnicas cuantitativas en las investigaciones académicas del derecho y las ciencias sociales es preocupante debido a la capacidad de generalización de los resultados que poseen estas técnicas, pues como se demostrará en esta reflexión, las técnicas cuantitativas como los números índice (NI) por medio de un solo número permiten a través de un conjunto de variables valorar y comparar en conjunto una categoría o unidad de análisis sin la necesidad de usar un método cualitativo, facilitando de esta manera la generalización de los resultados; así como también lo permite el método Análisis de contenido (AC) el cual puede demostrar el comportamiento de diferentes variables en un discurso oficial o político a lo largo de un periodo significativo mediante porcentajes de registro que señalan el grado de importancia que se les concede. En consecuencia, esta reflexión tendrá por fin: por un lado, indagar sobre las causas o razones que limitan un mayor uso de técnicas cuantitativas en las ciencias sociales y el derecho, de otro lado, ofrecer algunas experiencias en el uso de dos técnicas cualitativas que no son muy 
complicadas de aplicar: los números índice - $\mathrm{NI}$ - y el análisis de contenido -AC. El método usado es cualitativo y cuantitativo a la vez, con el uso de fuentes primarias y secundarias y la aplicación de técnicas como la entrevista, la revisión documental, los NI y el AC, para concluir finalmente las ventajas del uso de esta técnica en las investigaciones del derecho y las ciencias sociales. Uno de los hallazgos más relevantes es que el escaso uso de las técnicas cuantitativas obedece a un desconocimiento de estas herramientas por parte de los investigadores, antes que a una desconfianza o bajo interés sobre las mismas.

Palabras clave: metodología, metodología estadística investigación en ciencias sociales, investigación empírica, derecho.

\section{Abstract}

The diminished use of quantitative techniques in academic research of law and social sciences, is worrisome due to the generalizability of the results of these techniques As will be demonstrated in this reflection, quantitative techniques such as $\mathrm{NI}$ by means of a single number allow, through a set of variables, to assess and compare a category or unit of analysis together without the need to use a qualitative method, facilitating this way the generalization of the results; as well as the AC method allows it, which can demonstrate the behavior of different variables in an official or political discourse over a significant period by means of registration percentages that indicate the degree of importance given to them. Consequently, this reflection will aim to: on the one hand, investigate the causes or reasons that limit a greater use of quantitative techniques in the social sciences and law and, on the other hand, offer some experiences in the use of two qualitative techniques that do not they are very complicated to apply: index numbers - NI- and content analysis - AC. Both qualitative and quantitative method were used with primary and secondary sources and research techniques such as interview, documentary review, NI and AC, to finally conclude the advantages of using this technique in law and social science research. One of the most relevant finding is that low use of quantitative techniques is due to ignorance of these tools by researchers, rather than a lack of confidence or interest in them.

Keywords: methodology, statistical methodology, social science research, empirical research, law.

\section{Introducción}

Una de las características en los trabajos de investigación en ciencias sociales y en el derecho es la baja propensión al uso de métodos y técnicas cuantitativas por parte de investigadores y estudiantes. Para estos últimos, y salvo el caso de la economía, es realmente extraño calificar o evaluar trabajos a nivel de pregrado, de maestría o doctorado que incluyan de manera explícita y elaborada técnicas cuantitativas para la producción y análisis de la información. En el caso de trabajos investigativos en ciencia política o derecho, la situación es más dramática al respecto. ${ }^{1}$

Lo anterior, es una limitante para mejorar el alcance y aplicabilidad de los hallazgos, los cuales 
quedan supeditados, la mayoría de las veces, a estudios que terminan en argumentaciones tautológicas o en círculos valorativos sobre el deber ser. En el mejor de los casos, se utilizan cifras estadísticas o informes provenientes de fuentes secundarias, para dar soporte a los objetivos y comprobación de hipótesis.

No se trata de que la investigación cualitativa sea de menor rigurosidad que la cuantitativa, pues al final, como se sabe, tanto la magnitud numérica como los complejos descriptivos son meras representaciones de la realidad (Feyerabend 2000); sin embargo, cuando se trata de crear generalizaciones o abstracciones (teoría), los métodos cuantitativos funcionan mejor dado que el dato numérico es por sí mismo una abstracción de la realidad. Sin embargo, debemos conceder que las teorías más abstractas y genéricas se expresan en fórmulas matemáticas como lenguaje universal (Colciencias, 2001), y no se debe olvidar que una de las debilidades de las ciencias sociales, ha sido su baja capacidad para construir teorías generales (Merton, 2003; Meneses, 2013).

Todo esto es bastante polémico y discutible sin lugar a duda. Se puede llegar a la conclusión que el uso de un determinado método depende del interés y objetivos de la investigación. Por ejemplo, el estudio de casos, cualitativo por natura, no está interesado en la generalización pero si en la comprensión profunda de la situación (Mardones, 2006; Habermas, 1967).

\section{Metodología}

El objetivo de esta reflexión es doble: por un lado, se trata de indagar sobre las causas o razones que limitan un mayor uso de técnicas cuantitativas en las ciencias sociales y el derecho, de otro lado, ofrecer algunas experiencias en el uso de dos técnicas cualitativas que no son muy complicadas de aplicar: los números índice -NI- y el análisis de contenido -AC-.

Frente al primer aspecto, se pueden esgrimir a manera de hipótesis las siguientes razones que desestimulan el uso de técnicas cuantitativas: 1) La desconfianza hacia los métodos cuantitativos, bajo la creencia que no son aplicables en ciencias sociales; 2) El poco interés sobre su uso como resultado de las discusiones epistemológicas antipositivistas, que condujeron a la apuesta crítica de construir métodos propios; 3) El desconocimiento sobre el uso y aplicabilidad de técnicas cuantitativas (Alvira, 2002).

Sobre el segundo objetivo, se busca ofrecer de manera didáctica cómo se pueden aplicar las técnicas de los NI y del AC para la obtención y sistematización de información. Se trata de un uso complementario o concurrente con otras técnicas de carácter cualitativo, que se usaron en dos diferentes investigaciones: la primera, NI en un trabajo de grado en Ciencia Política (2001) y la segunda AC, en uno de Derecho (2009).

La metodología utilizada en este artículo corresponde a un estudio explicativo, por cuanto se trata de encontrar las razones por las cuales se hace poco uso de técnicas cuantitativas en las investigaciones y trabajos académicos en las ciencias sociales y el derecho. Se emplean métodos tanto cualitativo y cuantitativo a la vez; ya que, por un lado, se realiza una interpretación sobre la manera cómo algunos investigadores califican el uso e importancia de las técnicas cuantitativas, por otro lado, se aplican técnicas cuantitativas como los NI y el AC. 
Las fuentes de información fueron primarias (específicamente las personas entrevistadas) y secundarias (libros, artículos y documentos de internet); finalmente, las técnicas de recolección de información fueron la entrevista, la revisión documental, los NI y el AC (Vanegas, et. al, 2010).

\section{El conocimiento, importancia y uso de técnicas cuantitativas}

La investigación cuantitativa se refiere de manera amplia, al uso de métodos estadísticos para recopilar y analizar información que permita conocer ciertas características o variables sobre un fenómeno o población que se está estudiando. Su origen se remonta al empirismo de los siglos XVII y XVIII sobre todo en Inglaterra y Alemania, cuando se aplicó la estadística al estudio de asuntos económicos, demográficos o sociales; tuvo un gran predominio durante el siglo XIX debido a la aspiración de equiparar las nacientes ciencias sociales con las ciencias naturales. A esta corriente positivista, se antepuso una interpretativista o hermenéutica que enfatizó en los sujetos, la pluralidad y los aspectos cualitativos. El realismo como perspectiva epistemológica y el uso de métodos holísticos, parece ser un puente entre las dos anteriores posturas (Hueso y Cascant, 2012).

Como se indicó en la introducción, la metodología cuantitativa ofrece algunas ventajas a la hora de generalizar y extrapolar los resultados y se considera entonces, que la investigación cuantitativa debe reunir los siguientes criterios: validez, generalidad, fiabilidad y replicabilidad (Hueso y Cascant, 2012).

Sobre las técnicas cuantitativas debemos diferenciar en primer lugar: a) Las técnicas de recopilación de información (o mejor, de producción de datos), como son, por ejemplo, la encuesta, el experimento, el análisis de contenido y la sociometría entre las más importantes (Beltrán Villava, 2000), y b) Las técnicas de análisis de la información, entre las que se destacan la estadística con todo su arsenal analítico (por ejemplo, la estadística descriptiva, el análisis paramétrico, no paramétrico y multivariado, los números índices, los coeficientes, etc.) (Fernández Sampieri et.al., 2014), y los modelos matemáticos, por otro lado. Aquí no entraremos a definir cada uno de estos conceptos, solo nos interesa precisar su nivel de conocimiento y de uso en la investigación social (Keohane, 1994).

\section{Percepción sobre el uso de técnicas cuantitativas en las ciencias sociales y el derecho}

Para tal fin, se realizaron entrevistas a seis (6) profesores provenientes de diversas disciplinas sociales dedicados a temas de investigación (un licenciado en ciencias sociales, dos abogados, un administrador de empresas, un politólogo y un economista).

Se diseñó una entrevista muy corta compuesta por cuatro preguntas abiertas, que permitiera aplicarla de manera rápida y espontánea, no dando tiempo a los entrevistados de preparar las respuestas, sino de contestar con su experiencia y conocimientos que tenía en el momento. Las preguntas fueron:

1) ¿Qué opinión tiene sobre el uso de técnicas cuantitativas en la investigación científica? 
2) ¿Qué técnicas cuantitativas conoce? (mencionarlas)

3) ¿Ha usado técnicas cuantitativas en sus investigaciones? ¿Cuáles?

4)¿Cree que el uso de técnicas cuantitativas es necesario en la investigación social? (ver tabla 1)

Tabla 1. Resultados de entrevistas

\begin{tabular}{|c|c|c|c|c|}
\hline $\begin{array}{l}\text { Preguntal } \\
\text { Entrevistado }\end{array}$ & 1 & 2 & 3 & 4 \\
\hline Lic. Ciencias Sociales & $\begin{array}{l}\text { Son necesarias en tanto el objetivo } \\
\text { lo exija, sin importar si tienen un } \\
\text { corte cualitativo o cuantitativo o de } \\
\text { ciencias sociales o físicas. }\end{array}$ & La encuesta & $\begin{array}{l}\text { Sí, tabulación de encuestas, } \\
\text { tabulación de datos. }\end{array}$ & $\begin{array}{l}\text { Si, en cuanto las estadisticas } \\
\text { puedan proyectar tendencias; lo } \\
\text { importante es que el investigador las } \\
\text { conozca. En Colombia científicos } \\
\text { sociales somos dados a "pasar por } \\
\text { alto". }\end{array}$ \\
\hline Entrevistado 2, & $\begin{array}{l}\text { Mientras provenga de fuente fiable, } \\
\text { me parecen bien; pues puede haber } \\
\text { sesgos. }\end{array}$ & $\begin{array}{l}\text { Me coge corto... econometría, } \\
\text { algunos que tienen en } \\
\text { sociología y toda la estadística. }\end{array}$ & $\begin{array}{l}\text { Sí, los datos obtenidos de } \\
\text { instituciones internacionales y } \\
\text { nacionales. }\end{array}$ & $\begin{array}{l}\text { Sí, son excelente recurso para } \\
\text { analizar causas/consecuencias, por } \\
\text { ejemplo, en el tema del delito. }\end{array}$ \\
\hline Administrador de empresas & $\begin{array}{l}\text { Dentro de la investigación aplicada, } \\
\text { los análisis cuantitativos son } \\
\text { indispensables; luego puede } \\
\text { enviarla a la investigación científica, } \\
\text { pero depende de la formación de } \\
\text { cada profesional, pues algunas } \\
\text { manejan más números como en mi } \\
\text { caso. }\end{array}$ & $\begin{array}{l}\text { Encuesta, } \\
\text { descriptiva, } \\
\text { analitica. }\end{array}$ & $\begin{array}{l}\text { Sí, casi en todas, la investigación } \\
\text { descriptiva, la investigación de } \\
\text { marketing, la mixta (cuali/cuanti), la } \\
\text { investigación documental basada } \\
\text { en la estadística e informes } \\
\text { económicos. }\end{array}$ & $\begin{array}{l}\text { No, porque se puede hacer } \\
\text { investigación social desde la } \\
\text { sociología, antropología } \\
\text { educación, con técnicas de } \\
\text { observación cualitativa o entrevistas. }\end{array}$ \\
\hline Entrevistado 4, & $\begin{array}{l}\text { Que son oportunas, determinadas a } \\
\text { llegar a la generalidad de una } \\
\text { población; pueden originar un } \\
\text { sesgo informativo de acuerdo a la } \\
\text { aplicación o cobertura de una } \\
\text { política pública o los resultados de } \\
\text { aplicación de una ley. }\end{array}$ & $\begin{array}{l}\text { Muestreo poblacional, sondeo, } \\
\text { encuesta directa, las } \\
\text { estadisticas (medición de la } \\
\text { moda, media, varianza, } \\
\text { covarianza. }\end{array}$ & $\begin{array}{l}\text { En alguna oportunidad, una } \\
\text { encuesta, para indagar sobre el } \\
\text { término democracia y derechos } \\
\text { fundamentales. }\end{array}$ & $\begin{array}{l}\text { Depende del fin de la investigación; si } \\
\text { se requiere conocer el punto de vista } \\
\text { de una mayoría o comunidad. Pero } \\
\text { en general, es más para ciencias } \\
\text { naturales. }\end{array}$ \\
\hline Entrevistado 5 & $\begin{array}{l}\text { Son necesarias, incluso en } \\
\text { ciencias, pues no solo datos } \\
\text { cualitativos son suficientes. }\end{array}$ & $\begin{array}{l}\text { Estadísticas, entrevistas con } \\
\text { preguntas cerradas y las } \\
\text { abiertas pueden tener } \\
\text { elementos } \\
\begin{array}{l}\text { comunes } \\
\text { cuantitativos; la triangulación. }\end{array}\end{array}$ & $\begin{array}{l}\text { Sí, las anteriores: Estadísticas, } \\
\text { entrevista, triangulación. }\end{array}$ & $\begin{array}{l}\text { Sí, son necesarias. Son adecuadas } \\
\text { para obtener datos más reales y } \\
\text { objetivos sobre una situación } \\
\text { determinada. }\end{array}$ \\
\hline Entrevistado 6, & $\begin{array}{l}\text { Las técnicas cuantitativas por una } \\
\text { parte, son un apoyo metodológico a } \\
\text { la investigación, por otra, ayudan a } \\
\text { ratificar lo que afirma una teoría }\end{array}$ & $\begin{array}{l}\text { Estadística, probabilidades, } \\
\text { econometría (series de tiempo) } \\
\text { datos de panel. }\end{array}$ & $\begin{array}{l}\text { Sí, Correlaciones y estadísticas } \\
\text { básicas. }\end{array}$ & $\begin{array}{l}\text { Desde luego. Esto ayuda a realizar } \\
\text { trabajos más robustos en este } \\
\text { campo; por otra parte, ayuda a } \\
\text { superar las limitaciones que los } \\
\text { investigadores sociales muestran en } \\
\text { este tipo de investigaciones. }\end{array}$ \\
\hline
\end{tabular}

Frente al análisis de las entrevistas realizadas como respuesta a los interrogantes planteados se destacaron las siguientes respuestas.

1) ¿Qué opinión tiene sobre el uso de técnicas cuantitativas en la investigación científica?

Las diferentes respuestas a esta pregunta denotan una consideración positiva sobre las técnicas cuantitativas, por parte de los entrevistados. Para dos de ellos son necesarias, para otro son oportunas, otro las considera incluso indispensables en la investigación aplicada. Sin embargo, dos de los entrevistados advierten sobre los sesgos a que pueden llevar si no se manejan adecuadamente. También cabe señalar, que se las relaciona con la generalidad y la 
creación de teorías como en el caso de los entrevistados 3, 4 y 6.

2) ¿Qué técnicas cuantitativas conoce? (mencionarlas).

La encuesta, la estadística y la econometría parecen ser en su orden, las técnicas más conocidas por los entrevistados; también hubo referencias a las probabilidades y la triangulación. El entrevistado No. 5 mencionó la entrevista, la cual es considerada como técnica eminentemente cualitativa. Por otro lado, no hay una discriminación entre técnicas de captura y técnicas de análisis de la información.

3) ¿Ha usado técnicas cuantitativas en sus investigaciones? ¿Cuáles?

Prevalece el uso de la estadística (cuatro entrevistados), hay dos menciones a la encuesta y dos a la utilización de informes (con estadísticas); uno se refiere a la entrevista y otro a la triangulación como técnicas cuantitativas, lo cual no deja de sorprender esta confusión. En general, el uso de técnicas cuantitativas es más reducido que el conocimiento de estas, ya de por sí limitado.

4) ¿Cree que el uso de técnicas cuantitativas es necesario en la investigación social?

Cuatro de las seis respuestas son afirmativas, esto significa que para la mayoría las técnicas cuantitativas deben ser incluidas en la investigación social, ya sea para obtener resultados más reales y objetivos, para proyectar tendencias o analizar causas/consecuencias; por otro lado, dos investigadores manifestaron su desacuerdo, uno señaló que no eran necesarias, pues para ello estaban las técnicas cualitativas y otro adujo que las técnicas cuantitativas eran más para ciencias naturales.

En el apartado de las conclusiones se realizará el comentario final sobre el uso de técnicas cuantitativas de acuerdo con las entrevistas realizadas.

\section{Los Números Índice -Ni-}

\section{Noción}

La técnica de los NI es un procedimiento que consiste en reducir varios guarismos o datos a un solo indicador (índice), para recoger el significado de un concepto o variable de una manera más completa a como lo harían sus componentes de manera aislada o fragmentaria. Se debe partir de la realización de una encuesta para la obtención de los datos brutos o porcentajes de frecuencia. El cálculo de NI es aplicable solo a preguntas donde existan factores de valoración en una escala ascendente o descendente del tipo: mejor-peor, bueno-malo, frecuenteinfrecuente, siempre-nunca, etc. Uno de estos NI, son los índices de satisfacción, que señalan el grado de valoración positiva de un conjunto de factores que conforman un conjunto de preguntas referidas a un mismo asunto o tema (variable).

La valoración o ponderación se realiza según el peso porcentual que se quiera atribuir a cada factor o criterio; esta da un mayor peso a los criterios favorables o que ofrecen satisfacción al concepto (variable) en cuestión. Se realiza otorgando el mayor valor posible (1.0) al factor 
más favorable, a su vez que elimina el más desfavorable (ponderándolo por 0.0) y distribuye de manera descendente las asignaciones restantes entre los otros factores $(0.75,0.5,0.25$ o 0.66 y 0.33 dependiendo del número de opciones de respuesta), luego se suman los coeficientes y se obtiene un número (Serna y Gómez, 1996). De esta forma, a cada pregunta se le puede atribuir un número de satisfacción y si varias preguntas hacen aparte de una variable, se suman obteniendo así, el índice de satisfacción de una variable.

\section{Aplicación a un estudio en particular a las Ciencias sociales y el Derecho}

Con el fin de constatar el avance de la democracia local a partir de la aplicación de la descentralización territorial durante el periodo 1986-2000, se realizó un estudio con un diseño metodológico tanto cualitativo como cuantitativo (Jiménez, 2001; Ortiz, 2016). La categoría de análisis fue la democracia local, a la cual se le asignaron cuatro variables:

- Apropiación y control político

- Participación

- Gestión local

- Cultura política

En cuanto a la técnica cuantitativa, se diseñó una encuesta para obtener datos sobre los cuales se calcularon números índice a las primeras tres variables. La encuesta se aplicó en tres municipios de Cundinamarca para un total de 775 encuestados, así: 74 en Choachí; 298 en Chía y 403 en Fusagasugá. Se realizó un tipo de muestreo probabilístico en la medida de lo posible, usando la técnica del muestreo aleatorio simple (cada individuo tiene la misma probabilidad de ser escogido). Un $80.2 \%$ de los encuestados vivía en zona urbana y un $19.2 \%$ en zona rural. El $51.7 \%$ eran hombres y $48.3 \%$ mujeres. Las preguntas se agruparon por cada variable y fueron cerradas, pues es la manera para aplicar números índices. Dentro de las preguntas cerradas las hubo de selección múltiple, dicotómicas y comparativas. A continuación, se presentan las preguntas sobre la variable Apropiación y Control Político, a manera de ejemplo. https://drive.google.com/ open?id=1EupnM1rqjBxvmCY6ynG1la3xJno001n5\&authuser=investigaciones\%40miugca.edu. co\&usp=drive_fs

Los resultados de la encuesta, según porcentajes de frecuencia en la variable analizada fueron (ver tabla 2):

Tabla 2. Resumen porcentajes de frecuencias en el total de la muestra 


\begin{tabular}{|c|c|c|c|c|c|c|c|c|c|}
\hline \multirow[t]{2}{*}{$\begin{array}{l}\mathbf{N}^{\circ} \\
\text { Pregunta } \\
\end{array}$} & \multicolumn{2}{|c|}{ obtenidos } & \multicolumn{2}{|c|}{ Criterios } & \multirow{2}{*}{$\begin{array}{l}y \\
c \\
\end{array}$} & \multicolumn{3}{|c|}{ porcentajes } & \multirow[b]{2}{*}{ g } \\
\hline & Si & No & $\mathbf{a}$ & b & & d & e & $f$ & \\
\hline \multicolumn{10}{|l|}{ Variable 1} \\
\hline 1.1. & & & 16.7 & 24.0 & 17.8 & 41.4 & & & \\
\hline 1.2. & 18.3 & 81.7 & & & & & & & \\
\hline 1.3. & & & 5.5 & 9.4 & 54.9 & 30.1 & & & \\
\hline 1.4. & 60.2 & 39.8 & & & & & & & \\
\hline 1.4.b. & & & 5.1. & 12.5 & 8.8 & 37.1 & 2.5 & 33.6 & \\
\hline 1.5. & 23.7 & 76.2 & & & & & & & \\
\hline
\end{tabular}

Fuente: adaptación de Jiménez (2001)

Resultados que según el cálculo de números índice por pregunta señalan que, en primer lugar, los porcentajes de cada respuesta se multiplican por una ponderación que varía entre el 100\% (factor más favorable) y el 0\% (factor más desfavorable); la asignación de ponderaciones varía según el número de criterios y el tipo de pregunta, por ejemplo, para las de selección múltiple: la respuesta "Siempre", se pondera con (1.0); "Casi siempre", se pondera con (0.66); "Algunas veces", se pondera con (0.33); "Nunca”, se pondera con (0.0). En segundo lugar, se calcula el índice de satisfacción de la pregunta, multiplicando el porcentaje de frecuencia hallado con la ponderación respectiva y sumando todos los resultados. El ejemplo con la pregunta 1.1, es el siguiente (ver tabla 3):

Tabla 3. ¿Considera Ud. que la manera de hacer política en el municipio ha cambiado favorablemente?

\begin{tabular}{lccr}
\hline Criterios & $\begin{array}{c}\text { \% de } \\
\text { frecuencia }\end{array}$ & Ponderación Satisfacción \\
\hline Mucho & 16,7 & 1 & 16.7 \\
Algo & 24 & 0,66 & 15.84 \\
Muy poco & 17,8 & 0,33 & 5,87 \\
Sigue igual & 41.4 & 0 & 0 \\
Índice de satisfacción de la pregunta & $\mathbf{3 8 , 4 1}$ \\
\hline
\end{tabular}

Fuente: adaptación de Jiménez (2001)

Por otro ladó, en relación con el cálculo de números índice por variable, se identifica que corresponde al promedio de índices de satisfacción de las preguntas que conforman cada bloque. Señalan el grado o nivel de aceptabilidad o valoración de cada variable. De esta manera, el índice de la variable Apropiación y control político se obtendría de la siguiente 
manera (ver tabla 4):

Tabla 4.Índice de satisfacción para la variable Apropiación y control político

\begin{tabular}{|c|c|}
\hline Pregunta No. & $\begin{array}{c}\text { Índice de } \\
\text { Satisfacción }\end{array}$ \\
\hline 1.1 & 38,41 \\
\hline 1.2 & 18.3 \\
\hline …......................... & 29.8 \\
\hline 1.4. ........ & 60.2 \\
\hline 1.4. b ...... & 35,7 \\
\hline $1.5 . \ldots \ldots$ & 23.72 \\
\hline Índice variable & 34,35 \\
\hline
\end{tabular}

De esta misma manera se calculan los índices para las demás preguntas y variables, pudiendo entonces llegar a un índice final o número único que refleje el resultado final de una categoría general (sería el promedio de los índices de las tres variables), en este caso, sería un índice sobre la democracia local.

Adicionalmente, es necesario elaborar una matriz para definir los niveles de satisfacción sobre los números encontrados; esto depende del nivel o "rasero" de satisfacción que el investigador quiera establecer en consideración con los aspectos históricos, culturales y a las posibilidades reales de los resultados (ver tabla 5):

Tabla 5.Matriz de niveles de satisfacción

\begin{tabular}{|c|c|c|c|c|c|}
\hline Criterio & Muy bueno & Bueno & Regular & Malo & Muy malo \\
\hline Rangos de índices & $80 \%$ y más & $79 \%$ a $60 \%$ & $59 \%$ a $40 \%$ & $39 \%$ a $20 \%$ & $19 \%$ a 0 \\
\hline Intervalo & 20 & 20 & 20 & 20 & 20 \\
\hline $\begin{array}{l}\text { Nivel de } \\
\text { satisfagcjónglabo }\end{array}$ & ción nronia & $\begin{array}{l}\text { Bueno } \\
\text { sigutien }\end{array}$ & Aceptable & $\begin{array}{l}\text { Insuficiente } \\
\text { Serna v Gó }\end{array}$ & $\begin{array}{l}\text { Pésimo } \\
\text { pez (1996) }\end{array}$ \\
\hline
\end{tabular}

Con esta matriz se realiza una valoración cuantitativa y cualitativa acerca de los resultados obtenidos en cada variable y en cada entidad o caso analizado. Siguiendo con el ejemplo, la variable de la democracia local Apropiación y control político, obtuvo un índice de 34.35, que lo ubica como resultado "Malo" y con un nivel de satisfacción "Insuficiente"

Además del resultado matemático que deriva en una interpretación valorativa (en la cual no puede estar ausente el referente cualitativo al establecer el nivel de satisfacción), se pueden 
realizar análisis comparativos con los NI obtenidos en la misma variable pero en otro municipio, lo que permite enriquecer los resultados que se obtienen. Finalmente, se pueden comparar los resultados finales de todas las variables en cada municipio o caso estudiado, como se muestra a continuación (ver graficas 1 y 2):

Gráfica 1. NI en Apropiación y control político

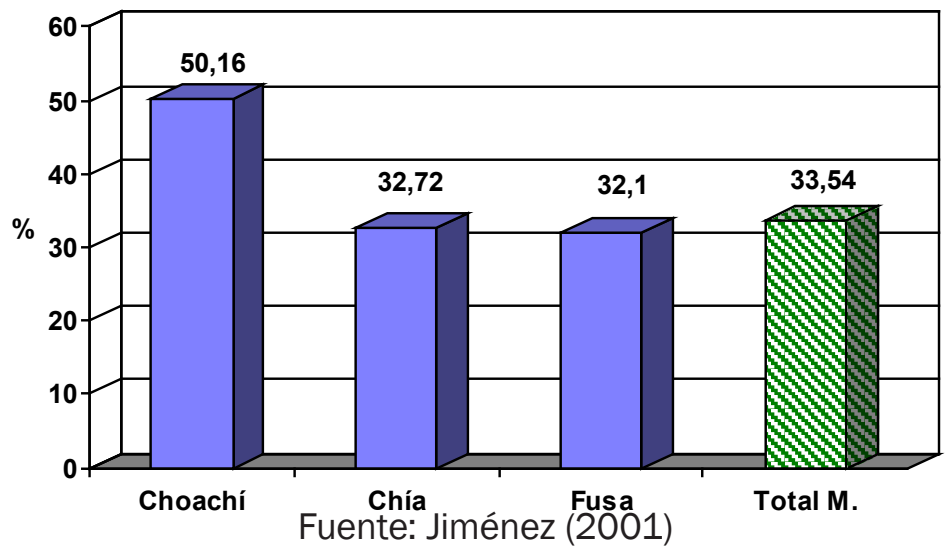

Gráfica 2. NI finales en todas la variables de los tres municipios

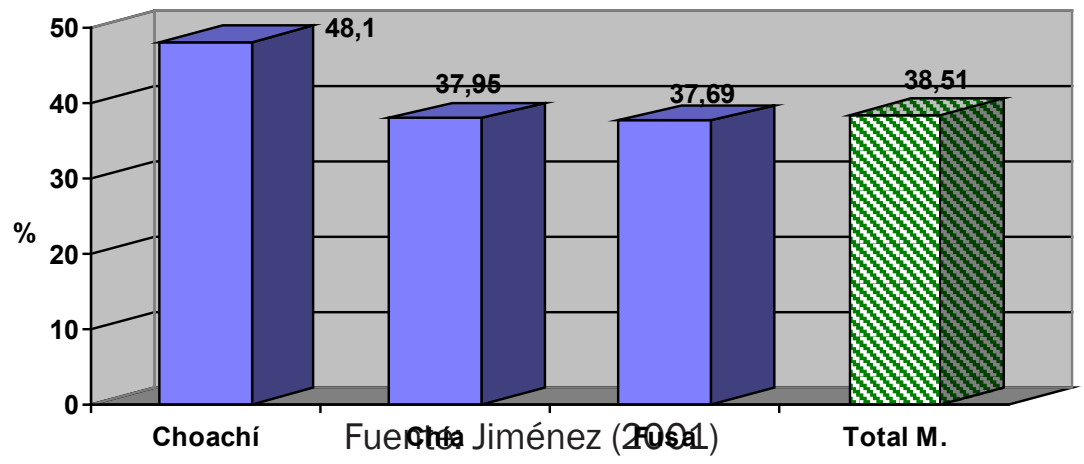

Gráfico que entonces dentro de este supuesto, representa que Choachí pasa el examen sobre el índice del total conglomerado, sus índices de Gestión pública y Apropiación y control político repercuten favorablemente en su evaluación, por lo que es el municipio con mejor calificación. El nivel de desarrollo de la democracia local, según el nivel de satisfacción de sus habitantes, es aceptable para el caso de Choachí.

El análisis de contenido -AC- 


\section{Noción}

Según Colle, el AC: “[...] es el conjunto de los métodos y técnicas de investigación destinados a facilitar la descripción sistemática de los componentes semánticos y formales de todo tipo de mensaje, y la formulación de inferencias válidas acerca de los datos reunidos" (s.f, p.1). En este sentido, es una técnica de producción de información típicamente cuantitativa, aunque puede ser usada de forma cualitativa (Beltrán Villalva, 2000). Para Shapiro y Markoff (citados en Colle, s.f. p 1), el AC se define como una “[...] reducción sistemática del flujo del texto (u otros símbolos) a un cuerpo estándar de símbolos manipulable estadísticamente, tal que represente la presencia, la intensidad o la frecuencia de ciertas características relevantes para la ciencia social". El AC tiene una metodología, unas modalidades y unas técnicas de procedimiento. Se consideran seis pasos para el AC:

a) Selección de las unidades de muestreo. Es aquella información que será estudiada, que tiene forma física y que constituye la realidad investigada (periódicos, libros, artículos, discursos, textos jurídicos, exposiciones de motivos, ponencias, etc.); a su vez, cada unidad de muestreo requerirá por lo menos, una plantilla o ficha para el registro de la información.

b) Selección de las categorías de análisis. Para el caso estudiado, estas categorías se refieren a la relación entre el derecho y el desarrollo, específicamente el derecho como norma jurídica (régimen jurídico territorial) y el desarrollo local.

c) Selección de unidades de registro. Son las partes analizables en que se divide una unidad de muestreo o los segmentos de contenidos que pueden ser categorizados, medidos o descritos de la misma manera sin destruir sus posibles relaciones con otras unidades de registro de una misma unidad de muestreo. En nuestro caso, un Plan Nacional de Desarrollo es una unidad de muestreo, que se divide en varias unidades de registro, por ejemplo, la introducción, cada uno de los capítulos y los presupuestos plurianuales.

d) Determinación de las unidades de análisis. Son los conceptos o ideas que se analizan dentro de las unidades de registro y constituyen la parte fundamental del trabajo de AC; se definen en el marco teórico del estudio y pueden presentarse explícitamente en el contenido (mediante un término o palabra asociada) o implícitamente (se encuentra el concepto, el sentido o significado, pero no la palabra o término). En nuestro caso, las unidades de análisis son tres: 1) Desarrollo local, 2) Autonomía local y 3) Descentralización territorial.

e) Determinación de las variables. Atribuyen caracteres, propiedades o rasgos a las unidades de análisis, facilitando su aprehensión y comprensión; hay que releer y registrar datos internos sobre variables para poder llegar a determinar una unidad de análisis. Existen variables abiertas (que permiten al analista escribir cualquier respuesta en el espacio prefijado) y variables cerradas (que han de prever todas las alternativas posibles); también las hay nominales y ordinales, aritméticas, implícitas y explícitas, etc. En el estudio antes propuesto, las variables son abiertas y definidas implícitamente debido a cierto margen que pueden significar o abarcar (ver tabla 6). Las unidades de análisis y sus variables son las siguientes:

- Desarrollo local: presencia/ausencia y manejo de conceptos relacionados con desarrollo 
económico local, desarrollo político-institucional local, desarrollo cultural y empoderamiento de la comunidad local, perspectiva medioambiental local y desarrollo tecnocientífico local.

- Autonomía local: presencia/ausencia y manejo de conceptos tales como autoridades propias, poder autorregulativo, recursos fiscales propios y administración de asuntos propios.

- Descentralización territorial: Presencia/ausencia de conceptos relacionados con el traspaso de competencias de nivel nacional a sus entidades territoriales en los aspectos: políticos, fiscal-financieros y administrativos.

e) Selección de las modalidades para la medición y evaluación. Hay dos grandes modalidades:

1) Modalidad cuantitativa o extensiva, que se utiliza cuando la cantidad de material es grande y se quiere obtener una visión de conjunto para efectuar comparaciones (datos estadísticos, frecuencias y sus relaciones); 2) Modalidad cualitativa o intensiva, que busca más el contenido latente, encontrar más detalles al evaluar una o varias dimensiones del discurso o del texto; el número de casos es más limitado (Piñuel, 2002).

\section{Aplicación a un estudio en particular en el derecho}

La técnica del AC, se utilizó en una investigación en el campo del derecho, cuyo objetivo general fue dilucidar el papel que ha tenido el derecho como conjunto de normas para el fomento del desarrollo local en los procesos de ordenamiento y descentralización territorial en Colombia, durante el periodo 1986-2007 (Jiménez, 2009). Los objetivos específicos se ocupaban de: a) Establecer la concepción sobre desarrollo local, autonomía y descentralización territorial que subyacen en el ordenamiento jurídico territorial; b) Determinar el tratamiento dado a los conceptos de desarrollo local, autonomía y descentralización territorial en los diferentes Planes Nacionales de Desarrollo involucrados durante el periodo de estudio; c) Establecer la opinión de algunos expertos, académicos y funcionarios sobre la concepción del desarrollo, la autonomía y la descentralización en el régimen jurídico territorial colombiano, y la importancia que le atribuyen al mismo para efectos del desarrollo local.

El diseño metodológico esencialmente cualitativo introdujo, no obstante, la técnica AC en su doble aspecto cuali/cuanti. El AC se realizó sobre diversas clases de unidades de registro (7 planes nacionales de desarrollo -PND-, 5 actos legislativos, 2 informes de ponencia en la Asamblea Nacional Constituyente, y el texto aprobado (original) de la Constitución de 1991); para la mayoría de unidades de muestreo se utilizó una única plantilla. Aquí sólo se presenta como ejemplo el AC realizado sobre el Plan Nacional de Desarrollo 1982-1986 (ver tabla 6). 
Tabla 6. Plan Nacional de Desarrollo 1993-1986 “Cambio con Equidad”

\begin{tabular}{|c|c|c|c|c|c|}
\hline \multirow{2}{*}{\multicolumn{6}{|c|}{$\begin{array}{l}\text { Unidad de muestreo: Plan Nacional de Desarrollo } 1 \\
\text { Unidades de registro: Todo el documento del plan } \\
\end{array}$}} \\
\hline & & & & & \\
\hline Unidad de análisis & Var. 1 & Var. 2 & Var. 3 Var. 4 & $\begin{array}{l}\text { Tota } \\
\text { l }\end{array}$ & Interpretación \\
\hline $\begin{array}{l}\text { Desarrollo local: } \\
0 \text { alusiones al } \\
\text { término, } \\
3 \text { términos } \\
\text { asociados. }\end{array}$ & 2 & & 2 & 7 & $\begin{array}{l}\text { Utilizan el término desarrollo regional y desarrollo } \\
\text { institucional del municipio; este último, se refiere } \\
\text { a que mediante la planeación, asuman liderazgo } \\
\text { y se modernicen. }\end{array}$ \\
\hline $\begin{array}{l}\text { Autonomía local: } \\
0 \text { alusiones al } \\
\text { término, } \\
2 \text { asociados. }\end{array}$ & 1 & 3 & & 6 & $\begin{array}{l}\text { Se refieren a la autonomía regional. Significa } \\
\text { aumentar capacidad para que municipios mejoren } \\
\text { su desempeño en sus asuntos. Énfasis en } \\
\text { generar recursos propios. }\end{array}$ \\
\hline $\begin{array}{l}\text { Descentral. territorial: } \\
1 \text { asociado. }\end{array}$ & & 1 & 2 & 4 & $\begin{array}{l}\text { Uso indistinto; abarca tanto descentralización } \\
\text { territorial como la descentralización por servicios. } \\
\text { Mayor énfasis en traslado de competencias. }\end{array}$ \\
\hline Total & & & & 17 & \\
\hline
\end{tabular}

Fuente: Jiménez (2009)

En total hay 17 registros sobre las tres unidades de análisis, lo cual significa que el desarrollo local representa el $41.1 \%$, la autonomía local el 35.2\% y la descentralización el 23.5\% de los registros. Las columnas de las variables no se totalizan debido a que no se refieren a los mismos aspectos para cada una de las unidades de análisis.

En conclusión, este plan de desarrollo destaca el tema del desarrollo local, si bien lo concibe dentro de un ámbito más amplio como lo es el desarrollo regional. Los aspectos tenidos en cuenta sobre el desarrollo regional se refieren al fortalecimiento de la capacidad institucional y el fomento de la participación ciudadana. El anterior AC se realizó sobre otros 7 PND a lo largo del tiempo, lo cual sirvió para realizar un análisis no solamente estacional sobre la importancia de las variables de estudio en cada periodo de gobierno, sino el análisis comparativo que tuvieron en el discurso oficial a lo largo del tiempo. La gráfica 3 resume lo señalado.

Al comparar los comportamientos de las unidades de análisis a lo largo de los 7 planes nacionales de desarrollo (28 años), se observó un crecimiento sostenido en los registros sobre desarrollo_local desde el plan del presidente Samper (1994-1998), hasta alcanzar el máximo histórico con el plan del presidente Uribe Vélez (2006-2010); esto significa que el tema ha ido ganado importancia creciente desde entonces, si bien durante los años 80 tuvo también relevancia. La curva de la descentralización territorial, en cambio, es descendente a partir del plan 2002-2006, luego de registrar cifras altas y sostenidas durante los gobiernos de Gaviria (1990-1994), Samper (1994-1998) y Pastrana (1998-2002); con todo, es la unidad de análisis que en el total del periodo registra los mayores niveles de frecuencia en los registros, así como en su participación porcentual. En lo que toca con la autonomía_local, es la unidad de análisis con más bajos registros durante todo el periodo. Solo obtuvo niveles importantes con el gobierno de Belisario Betancur (1983-1986), y algún repunte con Gaviria (1990-1994) como resultado del proceso constituyente. 
Gráfica 3. Porcentajes de registros en los pnd

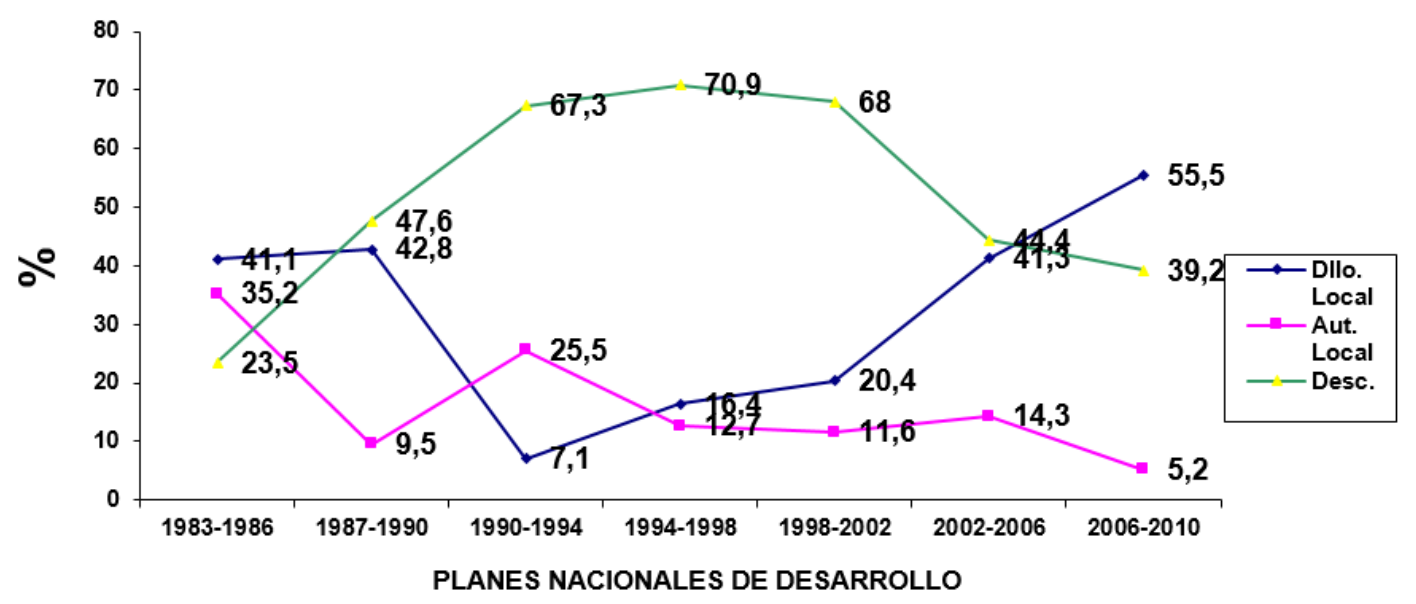

Fuente: Jiménez (2009)

\section{Conclusiones}

De acuerdo con las entrevistas realizadas -y aquí algo paradójico, que una técnica cualitativa sirva para establecer el uso de las técnicas cuantitativas-, se encontró que existe una opinión favorable hacia el uso de técnicas cuantitativas en la investigación científica y su necesidad en la investigación en las ciencias sociales y el derecho, pues si bien hay todavía cierta creencia acerca de que las técnicas cuantitativas son para las ciencias naturales y las cualitativas para las sociales. El conocimiento y uso de técnicas cuantitativas por parte de los entrevistados no parece ser muy bueno ya que, por un lado, se limitan al uso extendido de la estadística y algo de la encuesta y por otro lado, algunos se refieren equivocadamente al uso de la entrevista y la triangulación como aspectos cuantitativos.

Entonces, frente a las hipótesis señaladas al inicio de esta reflexión sobre las posibles razones por las cuales las técnicas cuantitativas se emplean poco en la investigación de las ciencias sociales y el derecho, los hallazgos señalan que esto se debe más al desconocimiento de las mismas, antes que por la desconfianza o poco interés que se tenga sobre ellas.

En el caso de los NI, se demostró cómo esta técnica a partir de la encuesta permitió asignar un solo número o dato a un conjunto de variables y de esta manera, a una categoría de análisis facilitando la generalización de los resultados para la construcción de teorías. Así, el nivel de satisfacción de la democracia local en los tres municipios estudiados es "insuficiente" (38.51\%), aunque muy cercana al nivel de "aceptable" (40\%) que se había determinado en la matriz de niveles de satisfacción. Esta sería la calificación final de la democracia local en los municipios colombianos obtenida gracias a los NI. 
Por su parte, el AC como herramienta cuantitativa, permite mostrar el comportamiento de ciertas variables de estudio, como en el ejemplo el desarrollo local, la descentralización y la autonomía territoriales en el discurso oficial de los Planes Nacionales de Desarrollo entre 1983 y 2010. La técnica logró capturar los porcentajes de registro sucesivos en los respectivos contenidos. Estos porcentajes (mayores o menores) señalan la importancia que el gobierno otorga a las variables estudiadas en el discurso, lo que posibilita completar el análisis con otros estudios de tipo financiero o presupuestal, para así determinar la correspondencia o no entre las declaraciones y las acciones.

Se recomienda entonces, el uso de técnicas cuantitativas en la investigación del derecho y ciencias sociales para poder tener un nivel de generalidad más sólido en los hallazgos y conclusiones; desafortunadamente, adolecemos de casos concretos en los cuales se haya aplicado o bien los $\mathrm{NI}$ o el AC en otras investigaciones jurídicas. A manera de ejemplo, en derecho privado (civil, comercial o financiero) se podrían realizar encuestas con preguntas cerradas o de escogencia de opciones como se presentó en los NI, para posteriormente reducir la información de diferentes preguntas o variables a un solo indicador o número índice; en cuanto al AC, este se podría aplicar a partir de analizar la exposición de motivos o la ratio decidendi de textos legales o jurisprudenciales durante un determinado periodo de tiempo, en los que previamente se hayan definido las variables (términos o palabras), y se procediera luego a establecer su ubicación, conteo y significado como se explicó en su oportunidad.

Finalmente, se recomienda el uso de técnicas cuantitativas junto con técnicas cualitativas. Lo ideal sería que en investigaciones propias del derecho y las ciencias políticas, los datos o la información fuera recopilada y analizadas a partir de estas dos técnicas para producir no solamente un efecto sinérgico, sino porque al final de todo, el dato numérico siempre requiere ser interpretado o valorado desde una perspectiva cualitativa.

\section{Referencias bibliográficas}

Alvira, M (2002). Perspectiva cualitativa / perspectiva cuantitativa en la metodología sociológica. México: Mc Graw Hill.

Beltrán Villava, M. (2000). Perspectivas sociales y conocimiento. Barcelona: Antrhopos.

Colciencias (2001). Guía para la elaboración de proyectos de investigación científica y tecnológica, Bogotá: Colciencias.

Colle, R. (s.f.). Análisis de Contenido.Recuperado de https://es.scribd.com/ document/123999172/analisis-contenido1, octubre de 2017.

Fernández Sampieri, et.al. (2014). Metodología de la Investigación, México, Mc Graw Hill, 6 edición.

Feyerabend, P (2000). Diálogo sobre el método. Madrid: Cátedra.

Habermas, J. (1967). La lógica de las ciencias sociales. Madrid: Tecnos. 
Hueso, A. y Cascant, J. (2012). Metodología y Técnicas Cuantitativas de Investigación, Cuadernos Docentes en Procesos de Desarrollo, n. ${ }^{\circ}$ 1, Valencia: Universitat Politécnica de Valencia. Recuperado de https://riunet.upv.es/bitstream/handle/10251/17004/ Metodología\%20y\%20técnicas\%20cu antitativas\%20de\%20investigación 6060.pdf. Consulta abril de 2021.

Jiménez, W. (2009). Derecho y desarrollo. Papel de la regulación jurídica en el desarrollo local, Bogotá: Universidad Católica de Colombia.

Jiménez, W. (2001). Democracia local y autonomía. Falsas expectativas y promesas incumplidas de la descentralización, Bogotá: ESAP .

Keohane, Robert (1994). El diseño de la investigación social : la inferencia científica en los estudios cualitativos. Madrid: Alianza.

Mardones, J. (2006). Filosofía de las ciencias humanas y sociales. Bogotá: Anthropos.

Meneses, O. (2013). “El derecho en el proceso de la civilización. Consideraciones metodológicas", en Criterio jurídico garantista. Año 5, No. 9, pp. 12-39.

Merton, R. (2003). Teoría y estructura sociales. México: FCE.

Ortiz, F.(2016).Diccionario de metodología de la investigación científica. México: Limusa.

Piñuel, J. L. (2002). Epistemología, metodología y técnica del Análisis de Contenido.Recuperado de http://anthropostudio.com/wp-content/uploads/2015/04/Jos\%C3\%A9-LuisPi\%C3\%B1uel-Raigada.-Epistemolog\%C3\%ADa-metodolog\%C3\%ADa-y-t\%C3\%A9cnicasdel-an\%C3\%A1lisis-de-contenido..pdf. Consulta octubre de 2017.

Serna, H. y Gómez, J. (1996), Auditoría del Servicio. Bogotá: RAM editores.

Vanegas, G, et. al. (2010). Guía para la elaboración de proyectos de investigación. Bogotá: Universidad Libre. 